\title{
Performance of Rapid Immunochromatographic Test on Malaria Infecton Among Expectant Women in Kericho County, Kenya
}

\author{
Annastasia Njeru, MSc \\ Joshua Mutiso, PhD \\ Department of Zoological Sciences, Kenyatta University, Nairobi, Kenya \\ Albert Kimutai, PhD \\ Department of Biological Sciences, University of Kabianga, Kericho, Kenya \\ Erick K Mibei, PhD
}

Kapkatet Campus, University of Kabianga, Kericho, Kenya

Doi: 10.19044/esj.2019.v15n6p330 URL:http://dx.doi.org/10.19044/esj.2019.v15n6p330

\begin{abstract}
Background: Microscopy is the gold standard method currently acceptable in diagnosis of malaria in a clinical setting but is subject to diagnostic bias as it cannot detect malaria parasites as they are very low or absent in peripheral circulation. Rapid immunochromatographic test (ICT) can detect the parasite antigen in peripheraral circulation and thus may be a suitable complementary epidemiological tool for malaria diagnosis among the expectant women in low parasitaemia areas. However, the sensitivity and accuracy of rapid ICT in diagnosis of malaria in pregnancy has not been established. Methods: A laboratory based cross sectional study was used. Blood samples from 314 pregnant women attending the antenatal clinic were screened for malaria parasites using microscopy, rapid ICT and polymerase chain reaction (PCR). Results: Out of the 314 samples analyzed for malaria, 44 samples $(14.0 \%)$ were positive for P. falciparum by microscopy while 62 (19.7\%) were positive by rapid ICT. The sensitivity and specificity of rapid ICT was $84.4 \%$ and $96.8 \%$ respectively while that of microscopy was $68.8 \%$ and $100.0 \%$ respectively. There was a moderate level of agreement between results of rapid ICT and microscopy and high level of agreement when rapid ICT results were compared against microscopy and PCR results $(\kappa=0.517 ; p$ $<0.001$ and $\kappa=0.821 ; \mathrm{p}<0.001)$ respectively. Conclusion:The findings of this study suggest the potential adoption of rapid ICT as a complementary epidemiology tool for malaria diagnosis among expectant women in areas of low parasitaemia and where microscopy is difficult to implement.
\end{abstract}


Keywords: Malaria, pregnancy, immunochromatographic, polymerase chain reaction, microscopy

\section{Introduction}

Despite the significant decline in Plasmodium falciparum infection, malaria still remains a major threat to pregnant women and children aged less than 5 years (WHO, 2015). Globally, 200,000 deaths occur among the infants and 10, 000 deaths among pregnant women annually (Desai et al., 2007). Over $25 \%$ of pregnant women in stable transmission areas of Africa are infected with malaria and an estimate of $50 \%$ of these women have positive parasitemia without their knowledge often in their placenta (Steketee et al., 2001). According to ACTwatch Group (2017) 70\% of population in Kenya is at risk of Malaria infection. However, substantial reductions in malaria mortality cases has been recorded between year 2010 and 2015 (NMCP, 2015). This decline is due to employment of malaria control interventions through provision of affordable artemisinin-based combination therapy (ACT) and increased availability of rapid ICT to enhance early detection, accurate diagnosis and prompt treatment of malaria (President's Malaria Initiative 2016; PSI, 2017).

Previous studies have reported that pregnant women are three times more likely to suffer from severe diseases attributed to malarial infection due to Plasmodium placental infestation compared to non-pregnant counterparts (WHO, 2006). This includes maternal anaemia, pre-term and low birth weight (Cottrell et al., 2015, Rogerson et al., 2018). World Health Organization has recommended the provision of insecticide-treated mosquito nets (ITNs) to pregnant women, intermittent preventive therapy in pregnancy (IPTp) as part of antenatal care and malaria screening for early detection and treatment during their first antenatal visit (WHO 2012). Inspite of these efforts, pregnant women are still highly vulnerable to malaria and high mortality rate arise from severe malaria. Therefore, there is need to improve the performance of malaria diagnostic techniques which is substantial in malaria reduction due to access to timely and appropriate treatment. Understanding the performance of rapid ICT and microscopy in the present study is thus very crucial.

Microscopy which is the gold standard method for identifying malaria infection has so many draw backs and alone may not be a suitable diagnostic tool in effective malaria control. It requires high proficiency to get accurate results since malaria parasites can be sequestered in the placenta (Umbers et al, 2011). It is also labour intensive and time consuming and thus not suitable in areas that report high cases of malaria and have limited resources. In addition, lack of skilled technologists in medical facilities in high transmission areas often leads to poor interpretation of data (Steketee 2001, NMCP, 2011, 
Abdul-Ghani et al 2015, Strom et al, 2013, Wilson, 2013). As a result, World Health Organization has recommended the use of rapid ICT in diagnosis of malaria inorder to promote the control of the infection (WHO 2014). However, their accuracy in detection of malaria among the pregnant women need to be investigated fully.

According to Dondorp et al.,(2011), this conventional technique has low sensitiviy to detect all the parasites of $P$ falciparum due to sequestration in the placenta during pregnancy (Goldring, 2004, Poespoprodjo et al 2008). Therefore, there is great need to develop a more sensitive diagnostic tool able to detect malaria parasites in peripheral circulation that remain undetectable through microscopic examination.

Previous studies in Uganda and Congo reported better performance of rapid ICT as compared to microscopy in detection of parasite antigen in peripheral circulation irrespective of sequestration of the parasites in the placenta (Kyabayinze et al, 2011; Matangila et al, 2014). However, the sensitivity and accuracy of rapid ICT in diagnosis of malaria among expectant women under different ecological areas have not been fully established. Therefore, this study evaluated the performance of rapid ICT as a complementary tool in diagnosis of malaria among expectant women in Kericho County, Kenya.

\section{Methods}

\section{Study design and patient population}

The study was a laboratory based cross sectional study utilizing immunochromatographic test for rapid diagnosis of malaria among antenatal women in Kericho County Referral Hospital and Kipsitet Health Centre in Kericho County. Kericho is located 264 km from Nairobi Kenya and lies $\left(0^{\circ} 22^{\prime} 8.03 " \mathrm{~S}, 35^{\circ} 17^{\prime} 10.63 " \mathrm{E}\right)$.

The study sites were selected through purposive sampling method. These centers have limited resources, rely on microscopy in diagnosis of malaria and have an antenatal care (ANC) clinic which record high antenatal clinic visits. The rainfall pattern in the area is bimodal with long rainy season between April and June and short rains from November to December.

\section{Enrolment of study participants}

The study involved all expectant women aged 18 years and above attending the antenatal clinic during their routine visit. The women were recruited as they entered the laboratory for malaria screening as recommended by the clinician and who were not actively on anti-malarial drugs. After informed consent, a brief structured questionnaire was used to collect information on parity and age. Five milliliters of blood was collected from participants and put in EDTA-coated bottles for malaria diagnosis using 
microscopy, rapid ICT and polymerase chain reaction (PCR). Blood samples collected for molecular analysis at Kericho District Hospital and Kipsitet Health Centre were analyzed at Kenya Medical Research Institute Laboratories, Nairobi. This study was reviewed and approved by Moi Teaching and Referral Hospital (MTRH)-Moi University IREC and by Kericho County Referral Hospital Research and Training Committee. Informed consent was obtained from all participating expectant women before recruitment into the study.

\section{Determination of parasitaemia level by microscopy}

Thick blood films were prepared by placing a drop of blood on a slide, spreading it in a circular motion and allowing it to air dry. The slides were then stained for 10 minutes using $10 \%$ Giemsa stain, washed in distilled water and allowed to dry in a vertical position. The slides were evaluated using light microscopy (100X oil immersion lenses) for malaria by two independent microscopists and the results were blinded. Discrepancy in results between the two microscopists was resolved by re-reading of the slides by a third microscopist before the final results were determined. Parasitaemia level was calculated by counting malaria parasites in 200 leukocytes and multiplying the resulting value by 40 assuming that each microliter of blood contain 8,000 leukocytes as described by WHO (2015). Any discrepancy in parasite count readings was resolved by computing the mean of the parasitaemia reading by the two microscopists. If no parasite was recorded after counting 200 leucocytes, a slide was then considered as negative.

\section{Rapid Immunochromatographic Test}

The Rapid ICTs kit used in this study was SD Bioline 05FK60 One Step Malaria Antigen P. falciparum / Pan-test (Standard Diagnostic Inc.) and procedure for testing was done as described by the manufacturer. Briefly, using a capillary tube, $5 \mu \mathrm{L}$ of whole blood was drawn from the EDTA bottle and transferred into a round sample well followed by 4 drops of assay diluent which were placed into a square well. The test results were read after 15 minutes. The test was considered positive if a band was formed at both test and control lines and negative if the band formed at the control line only.

\section{Polymerase Chain Reaction assay}

Three drops of blood were spotted directly on $3 \mathrm{~mm}$ Whatman's filter paper at different points, allowed to air dry, then placed individually in plastic bags and stored at $-20^{\circ} \mathrm{C}$ with a silica gel desiccant to prevent dampness for DNA extraction. Extraction of parasite DNA from filter papers was performed using Chelex-100 method as described by Hsiang et al., (2010). Briefly, the piece of filter paper was transferred to a sterile $1.5 \mathrm{ml}$ microfuge tube 
containing $1 \mathrm{ml}$ of $0.5 \%$ saponin in $1 \mathrm{X}$ phosphate buffered saline (PBS) and incubated overnight at $4^{\circ} \mathrm{C}$. The brown solution was discarded and replaced with $1 \mathrm{ml}$ phosphate buffered saline and incubated at $4^{\circ} \mathrm{C}$ for 30 minutes. The brown solution was also discarded and $50 \mu \mathrm{l}$ of stock $20 \%$ Chelex solution added to $150 \mu \mathrm{l}$ of DNase-free water in a $0.5 \mathrm{ml}$ microfuge tube containing the filter paper. The component in the tube was heated at $100^{\circ} \mathrm{C}$ for 2 minutes followed by vortexing and this was done for a total of 10 minutes. The tube was then centrifuged at $10000 \times \mathrm{g}$ for 2 minutes and then the supernatant transferred to another fresh tube and centrifuged for another 2 minutes. The final supernatant was transferred into another fresh tube to be used as a DNA template. The amplification of K-13 propeller gene was done on MJ Thermocycler ${ }^{\mathrm{TM}}$ PCR machine.

The PCR reaction involved outer and nested reaction in order to enhance specificity. One microlitre of DNA extracted through Chelex method was used as a template in outer PCR amplification and was added into a reaction mixture of $29 \mu \mathrm{L}$ containing $3 \mu \mathrm{L} 10$ X PCR buffer, $3.6 \mu \mathrm{L} 25 \mathrm{mM}$ Magnesium Chloride, $0.3 \mu \mathrm{L} 20 \mathrm{mM}$ dNTPs, $0.3 \mu \mathrm{L}$ of each of $10 \mu \mathrm{M}$ primer (K13-1 and K13-4), 1.2 $\mu \mathrm{L}$ TaqDNA polymerase $(5 \mathrm{u} / \mu \mathrm{L})$ and $20 \mu \mathrm{L}$ of double distilled water hence making up a total volume of $30 \mu \mathrm{L}$. The K13propeller domain was amplified using, K13-1 5'CGGAGTGACCAAATCTGGGA-3'and K13-4 5'GGGAATCTGGTGGTAACAGC-3' as forward and reverse primers.

Thermocycling conditions were $94^{\circ} \mathrm{C}$ for 5 minutes, followed by 40 cycles of $94^{\circ} \mathrm{C}$ for 30 seconds, $60^{\circ} \mathrm{C}$ for 90 seconds, $72^{\circ} \mathrm{C}$ for 90 seconds and a final extension of $72^{\circ} \mathrm{C}$ for 10 minutes. Two microlitres of outer PCR product was used in nested PCR amplification and was added into a reaction mixture of $28 \mu \mathrm{L}$ containing $3 \mu \mathrm{L} 10$ X PCR buffer, $3.6 \mu \mathrm{L} 25 \mathrm{mM}$ Magnesium Chloride, $0.3 \mu \mathrm{L} 20 \mathrm{mM}$ dNTPs, $0.3 \mu \mathrm{L}$ of each $10 \mu \mathrm{M}$ primer (K13-2 and K13-3), 1.2 $\mu \mathrm{L}$ TaqDNA polymerase $(5 \mathrm{u} / \mu \mathrm{L})$ and $19.3 \mu \mathrm{L}$ double distilled water thus making up a total volume of $30 \mu \mathrm{L}$. The forward and reverse primer sequences used for amplification of K13 propeller gene in nested PCR were K13-2 5'-GCCAAGCTGCCATTCATTTG-3' and K13-3 5' GCCTTGTTGAAAGAAGCAGA-3' respectively.

The thermocycling conditions for nested PCR were similar to outer PCR. For each run, DNA extracted from P. falciparum laboratory-adapted strain was used as positive control while water was used as negative control. Analysis of PCR products was done using $2 \%$ agarose gel electrophoresis and visualization done under UV light on a transilluminator using gel documentation system (VilbertLourmat). 


\section{Sensitivity, specificity and predictive values}

McNemar's $2 \times 2$ contingency table was used to calculate the sensitivity, specificity, positive and negative predictive values of rapid ICT (test under evaluation) using microscopy as the gold standard and polymerase chain reaction as a confirmatory test. The number of true positives (TP), number of true negatives (TN), number of false positives (FP) and number of false negatives (FN) were worked out. Sensitivity was calculated as TP/ (TP $+\mathrm{FN})$. Specificity was calculated as $\mathrm{TN} /(\mathrm{TN}+\mathrm{FP})$. The positive predictive value $(\mathrm{PPV})$ was calculated as $\mathrm{TP} / \mathrm{TP}+\mathrm{FP})$ and negative predictive value (NPV) was calculated as TN/ $(\mathrm{FN}+\mathrm{TN})$. Accuracy of the test, defined as proportion of all tests that gave a correct result, was calculated as (TP + $\mathrm{TN}) /$ number of all tests.

\section{Statistical analysis}

The coded data was entered on MS-Excel and imported into IBM SPSS Statistics v 22.0 (IBM Corp., Armonk, NY) for analysis. Assessment of the agreement between rapid ICT, microscopy and PCR was compared using Cohen Kappa Index.

\section{Ethical Approval}

Ethical Approval of the study was obtained from Moi Teaching and Referral Hospital/Moi University IERC (Formal approval No. FAN:IREC 1043). Additional approval was obtained from Ministry of Medical Services in charge of Kericho County where the study sites are located. Written informed consent was obtained before inclusion in the study. Only those who consented were allowed to participate in the study.

\section{Results}

\section{Prevalence of malaria based on Microscopy, rapid ICT test and PCR}

Table 1 summarizes results on the malaria prevalence among the sampled women based on the three diagnostic techniques that were utilized in the study. Overall, $62(19.7 \%)$ and $44(14.0 \%)$ women diagnosed as positive for malaria by rapid ICT test and microscopy respectively. Further, 20 of the 38 samples (52.6\%) subjected to the confirmatory test (PCR) were positive for malaria. Those who were found positive for malaria by both microscopy and PCR were 64 (20.4\%). 
Table 1: Malaria prevalence based on Microscopy, rapid ICT and Microscopy and PCR

\begin{tabular}{lllll}
\hline Test & \multicolumn{2}{c}{ Positive } & \multicolumn{2}{c}{ Negative } \\
\cline { 3 - 5 } & Number & \% & Number & \% \\
\hline Rapid ICT & 62 & 19.7 & 252 & 80.3 \\
Microscopy & 44 & 14.0 & 270 & 86.0 \\
Microscopy and PCR & 64 & 20.4 & 250 & 79.6 \\
\hline
\end{tabular}

ICT: Immuno-chromatographic test; PCR: polymerase chain reaction. Rapid ICT detected more positive samples otherwise missed by microscopy while PCR confirmed another two positive samples missed by microscopy

\section{Evaluation of the performance of rapid ICT and microscopy}

The sensitivity, specificity, positive predictive value (PPV), and negative predictive value (NPV) were computed for the rapid ICT. In the present study, malaria cases diagnosed using PCR and microscopy were regarded as the "true positives", else "true negatives". The sensitivity and specificity of rapid ICT was $84.4 \%$ (95\% confidence interval (CI) $73.6 \%$ $91.3 \%$ ) and $96.8 \%$ (95\% CI $93.8 \%-98.4 \%$ ) respectively while that of microscopy was $68.8 \%$ (95\% CI 56.6\% - 78.8\%) and $100.0 \%$ (95\% CI 98.5\% - 100.0\%) respectively. The overall diagnostic accuracy of rapid ICT was 94.3\% (95\% CI 91.1\% - 96.3\%) while that of microscopy was $93.6 \%$ (95\% CI $90.4 \%-95.8 \%$ ) as shown in Table 2 below.

Table 2: Assessment of validity of ICT and microscopy

\begin{tabular}{llcccccccc}
\hline Test & \multicolumn{2}{l}{ Sensitivity } & \multicolumn{2}{c}{ Specificity } & PPV & NPV & Accuracy \\
\hline & $(\mathbf{n = 6 4})$ & $\%$ & $(\mathbf{n}=\mathbf{2 5 0})$ & $\%$ & No. & $\%$ & No. & \% & \% \\
\hline Rapid ICT & 54 & 84.4 & 242 & 96.8 & 52 of 62 & 87.1 & 242 of 252 & 96.0 & 94.3 \\
Microscopy & 44 & 68.8 & 250 & 100 & 44 of 44 & 100 & 250 of 270 & 92.6 & 93.6 \\
\hline
\end{tabular}

n: sample size; PPV: positive predictive value; NPV: negative predictive value; Sensitivity indicates the probability that the test will be positive if malaria is present while specificity indicates the probability that the test will be negative if malaria is absent. Sensitivity was defined as the proportion of true positives (RDT or Light microscopy positive) from all matched positive qPCR samples while specificity was defined as the proportion of true negatives (qRDT or light microscopy negative results) of all matched qPCR negative samples. Positive predictive value was defined as how frequently the RDT or LM tested positive in matched qPCR positive samples, while negative predictive value was defined as the frequency of RDT or LM testing negative in matched PCR negative samples

\section{Level of agreement between rapid ICT, microscopy and microscopy and PCR}

Cohen's $\kappa$ was run to determine if there was agreement between rapid ICT, microscopy and microscopy and PCR in the diagnosis of malaria.. There was moderate level of agreement observed when microscopy was assessed against ICT $(\kappa=0.517 ; \mathrm{p}<0.001)$. Additionally, high level of agreement was 
observed between the results obtained from ICT and those obtained by a combination of microscopy and PCR $(\kappa=0.821 ; \mathrm{p}<0.001)$ (Table 3$)$.

Table 3: Assessment of the agreement between rapid ICT, microscopy and microscopy and PCR

\begin{tabular}{llllll}
\hline \multicolumn{7}{c}{ Rapid ICT Results } \\
\hline Test & $\begin{array}{l}\text { Total } \\
(\mathbf{n = 3 1 4})\end{array}$ & $\begin{array}{l}\text { Positive } \\
(\mathbf{n = 6 2})\end{array}$ & Negative (n=252) & K & P-value \\
\hline $\begin{array}{l}\text { Microscopy } \\
\text { Positive }\end{array}$ & 44 & $34(77.3)$ & $10(22.7)$ & 0.517 & $<0.001$ \\
$\begin{array}{l}\text { Negative } \\
\text { Microscopy and }\end{array}$ & 270 & $28(10.4)$ & $242(89.6)$ & & \\
$\begin{array}{l}\text { Positive } \\
\text { Negative }\end{array}$ & 64 & $54(84.4)$ & $10(15.6)$ & 0.821 & $<0.001$ \\
\hline
\end{tabular}

K: Cohen kappa index (k); n: sample size; ICT: immuno-chromatographic test; PCR: polymerase chain reaction. Assessment of performance agreement between RDT and Light Microscopy and Performance characteristics of RDT against qPCR for detection of infection by malaria parasite in peripheral blood samples was done where there was significant agreement between RDT and microscopy and PCR.

\section{Discussion}

This study evaluated the performance of rapid immnochromatographic test and microscopy in diagnosis of malaria among expectant women attending antenatal clinic in Kipsitet Health Centre and Kericho Distrit and Referral Rospital in Kericho County. Rapid ICT diagnosed significant higher number of positive cases recorded by rapid ICT compared to microscopy and this is attributed to its higher sensitivity. The higher sensitivity of rapid ICT compared to microscopy can be expected because during pregnancy, malaria parasites disappear from peripheral circulation and remain undetectable through microscopic examination due to sequestration of the parasites in the placenta (Goldring 2004, Desai et al., 2007, Cox-Singh et al., 2008). In contrast, parasite antigen is present in circulation and can be readily identified by rapid ICT (Goldring 2004, Fancony et al., 2013). The study findings cast doubt on the justification for use of microscopy as the gold standard method in diagnosis of malaria in pregnancy. The continued use is likely to excecerbate misdiagnosis and its unintended consequences.

Higher sensitivity and accuracy was recorded by rapid ICT compared to microscopy in the present study, thus revealing its reliability as a complementary tool in malaria diagnosis among expectant women in areas with low parasite density levels. Previous studies from Yemen, Congo, Angola, supports the ability of rapid ICT to detect malaria parasites below the threshold of microscopy (Harchut et al, 2013, Fancony et al., 2013, Alareqi et al., 2016). High level of agreement observed between the results obtained from rapid ICT and those obtained by a combination of microscopy \& PCR shows that rapid ICT can be used to complement microscopy in parasitological 
diagnosis of malaria among expectant women. In fact it has the potential to be adopted as the tool of choice in diagnosis of malaria especially in pregnancy where misdiagnosis has potential adverse effects for the mother and the unborn child. This was also demostrated by studies in Uganda and in Burkina Faso which suggested HRP2-based RDTs were the most appropriate diagnostic tests for use in expectant women (Kyabayinze et al., 2016).

The few malaria negative cases recorded by microscopic examination in the present study could be an indication of false negative result. This may be attributed to lack of technical expertise and unfamiliarity of laboratory personnel to malaria parasite. The presence of false negatives in microscopy diagnosis predisposes pregnant women suffering from malaria to poor maternal and foetal outcomes. The false positives from microscopic tests in the present study highlight the urgent need for additional training of laboratory personnel on microscopic diagnosis of malaria. This is particularly important in the tropics where the presence of other blood parasites and pathogens may interfere with accurate detection of malaria parasites (Kyabayinze et al, 2010, Bissofi 2011). The inability to perform effective diagnosis of malaria greatly increases indiscriminate use of antimalarials among pregnant women hence increasing rate of drug resistance (Hasting and Watkins, 2005).

Although pregnant women who were under antimalarial treatment 2 weeks before initiating the project and during the course of this study were excluded from the present study, false positives were recorded by rapid ICT using microscopy and PCR as a confirmatory test. However, this may have resulted because antigens can remain detectable in the blood stream several weeks after recovery from malaria (Lee et al, 2008, Hviid 2010, Kyabayinze et al, 2010; Iiombe et al.,2014). The performance of malaria rapid diagnostic kits is also affected by antigenic variation in Plasmodium falciparum where deletion of HRP2 gene is associated with false negatives results (Maltha 2013, Cheng et al. 2014, Ishengoma et al 2016, Kumar et al. 2013). However according to Global Malaria Programme report (2016), deletion of HRP2 gene is not usually the main cause of false negative malaria cases. This study did not determine the $P$. falciparum antigenic types present in the study population and thus the role of antigenic variation in detection of false negatives is not clear and should be the focus of future studies. Other causes of false positive and false negative results may be due to procedural aspects when conducting the tests and inappropriate storage temperatures. However, in the present study, the efficiency of rapid ICT was validated by formation of control band in all the tests. Therefore, there is need to investigate the factors that may lower the efficiency of this promising rapid technique in malaria diagnosis. In the present study rapid ICT performed better than microscopy and these findings are similar to what was reported in Angola (Fancony et al., 2013) and Brazilian Amazon (Andrade et al., 2010). 


\section{Conclusion}

Rapid ICT was found to have a significant higher sensitivity and accuracy in detection of Plasmodium falciparum in pregnancy hence proving to be a suitable and reliable alternative in diagnosis of malaria among expectant women in low malaria parasite density areas.

\section{Competing interests}

The authors declare that they have no competing interests.

\section{Funding}

This study was funded by a University of Kabianga Research Fund award.

\section{Authors' contributions}

AN carried out field work, laboratory analysis and drafted the manuscript. JM and AK contributed to the draft of the manuscript, EM conceived and designed the study and overall supervision of the study. All authors read and approved the final draft of the manuscript.

\section{Acknowledgments}

We would like to thank the expectant mothers who supported this study through their participation. We are grateful to the management of both Kericho County Referral Hospital and Kipsitet Health Centre for allowing this study at their respective institutions. We also acknowledge the contribution of the staff at the two institutions and the PCR team at KEMRI Nairobi. Finally, we are thankful to Ephantus J. Muturi for his assistance in data analysis and for his valuable comments on the manuscript.

\section{References:}

1. Abdul-Ghani R (2015). Research priorities in Yemen: paving the way for malaria elimination. Yemeni J Med Sci.9:1-5.

2. ACTwatch Group, Anne M, Waqo E, Rebecca K, Dorothy M and Edward A (2017). The malaria testing and treatment landscape in Kenya: results from a nationally representative survey among the public and private sector in 2016. Malar J. 16:494

3. Alareqi MQ, Mahdy A.K, Yee-Ling Lau, Mun-Yik Fong, Rashad Abdul

4. Ghani, Ali A et al., (2016). Field evaluation of a PfHRP-2/pLDH rapid diagnostic test and light microscopy for diagnosis and screening of falciparum malaria during the peak seasonal transmission in an endemic area in Yemen. Malar J. 15:49

5. Andrade BB, Reis-Filho A, Barros AM, Souza-Neto SM, Nogueira LL, Fukutani KF, et al., (2010). Towards a precise test for malaria 
diagnosis in the Brazilian Amazon: comparison among field microscopy, a rapid diagnostic test, nested PCR, and a computational expert system based on artificial neural networks. Malar J. 9:117.

6. Bisoffi Z, Sirima SB, Meheus F, Lodesani C, Gobbi F, Angheben A, et al., (2011). Strict adherence to malaria rapid test results might lead to a neglect of other dangerous diseases: a cost benefit analysis from Burkina Faso. Malar J. 10:226.

7. Cheng Q, Gatton ML, Barnwell J, Chiodini P, McCarthy J, Bell D, et al., (2014). Plasmodium falciparum parasites lacking histidine-rich protein 2 and 3: a review and recommendations for accurate reporting. Malar J.;13:283.

8. Cottrell G, Moussiliou A, Luty AJ, Cot M, Fievet N, Massougbodji A, et al., (2015). Submicroscopic Plasmodium falciparum infections are associated with maternal anemia, premature births, and low birth weight. Clin Infect Dis. 60:1481-8.

9. Cox-Singh, J., Davis, T. M., Lee, K. S., Shamsul, S. S., Matusop, A., Ratnam, S., Rahman, H. A., Conway, D. J. and Singh, B. (2008). Plasmodium knowlesimalaria in humans is widely distributed and potentially life threatening. Clin and Infect Dis 46: 165-171.

10. Desai, M., TerKuile, F. O., Nosten, F., McGready, R., Asamoa, K., Brabin, B. and Newman, R. D. (2007). Epidemiology and burden of malaria in pregnancy. Lancet Infect Dis 7: 93-104.

11. Dondorp, A. M., Fairhurst, R. M. and Slutsker, L. (2011). The threat of artemisinin resistant malaria. New England Journal of Medicine 365: 1073-1075.

12. Fancony C, Sebastiao YV, Pires JE, Gamboa D, Nery SV (2013). Performance of microscopy and RDTs in the context of a malaria prevalence survey in Angola: a comparison using PCR as the gold standard. Malar J.12:284

13. Global Malaria Programme (2016). False-negative RDT results and implications of new reports of $P$. falciparum histidine-rich protein $2 / 3$ gene deletions. Geneva: World Health Organization.

14. Goldring, J. P. D. (2004). Evaluation of immunotherapy to reverse sequestration in the treatment of severe Plasmodium falciparum malaria. J Immun and Cell Biol 82: 447-452.

15. Harchut, K., Standley, C., Dobson, A., Klaassen, B., RambaudAlthaus, C., Althaus, F. and Nowak, K. (2013). Over-diagnosis of malaria by microscopy in the Kilombero Valley, Southern Tanzania: an evaluation of the utility and cost-effectiveness of rapid diagnostic tests. Malar J 12: 159 doi: 10.1186/1475-2875-12-159.

16. Hastings IM, Watkins WM (2005). Intensity of malaria transmission and the evolution of drug resistance. Acta Trop. 94:218-29 
17. Hsiang, M. S., Lin, M., Dokomajilar, C., Kemere, J. and Pilcher, C. D. (2010). PCR-based pooling of dried bloodspots for detection of malaria parasites: optimization and application to a cohort of Ugandan children. J Clin Microbiol 48: 3539-3543.

18. Hviid, L. (2010). The role of Plasmodium falciparum variant surface antigens in protective immunity and vaccine development. Hum Vaccin6: 84-89.

19. Ilombe G, Maketa V, Mavoko HM, da Luz RI, Lutumba P, Van geertruyden JP (2014). Performance of HRP2-based rapid test in children attending the health centre compared to asymptomatic children in the community. Malar J.13:308.

20. Ishengoma DS, Shayo A, Mandara CI, Baraka V, Madebe RA, Ngatunga D, et al., (2016). The role of malaria rapid diagnostic tests in screening of patients to be enrolled in clinical trials in low malaria transmission settings. Health Syst Policy Res.3:2.

21. Kumar N, Pande V, Bhatt RM, Shah NK, Mishra N, Srivastava B, et al., (2013). Genetic deletion of HRP2 and HRP3 in Indian Plasmodium falciparum population and false negative malaria rapid diagnostic test. Acta Trop.125:119-21.

22. Kyabayinze DJ, Tibenderana JK, Nassali M, Tumwine LK, Riches C, Montague M, et al., (2011). Placental Plasmodium falciparum malaria infection: operational accuracy of HRP2 rapid diagnostic tests in a malaria endemic setting. Malar J.10:306.

23. Kyabayinze DJ, Zongo I, Cunningham J, Gatton M, Angutoko P, Ategeka J, Compaoré Y, Muehlenbachs A, Mulondo J, Nakalembe M, Somé FA, Ouattara A, Rouamba N, Ouédraogo J, Hopkins H, Bell D (2016). HRP2 and pLDH-Based Rapid Diagnostic Tests, Expert Microscopy, and PCR for Detection of Malaria Infection during Pregnancy and at Delivery in Areas of Varied Transmission: A Prospective Cohort Study in Burkina Faso and Uganda. PLoS ONE 11(7): e0156954. doi:10.1371/journal.pone.0156954

24. Kyabayinze, D. J., Asiimwe, C., Nakanjako, D., Nabakooza, J., Counihan, H. and Tibenderana, J. K. (2010). Use of RDTs to improve malaria diagnosis and fever case management at primary health care facilities in Uganda. Malar J 9: 200 doi: 10.1186/1475-2875-9-200.

25. Lee SW, Jeon K, Jeon BR, Park I. (2008). Rapid diagnosis of vivax malaria by the SD Bioline Malaria Antigen test when thrombocytopenia is present. J ClinMicrobiol, 46: 939-942.

26. Maltha, J., Gillet, P. and Jacobs, J. (2013). Malaria rapid diagnostic tests in endemic settings. JClinMicrobiol and Infect Dis 19: 399-407.

27. Matangila J, Lufuluabo J, Ibalanky A, Inocencio da Luz R, Lutumba P Van Geertruyden J (2014). Asymptomatic Plasmodium falciparum 
infection is associated with anaemia in pregnancy and can be more cost-effectively detected by rapid diagnostic test than by microscopy in Kinshasa, Democratic Republic of the Congo. Malar J.13:132.

28. National Malaria Control Programme (NMCP) and ICF International (2015). Kenya Malaria Indicator Survey.

29. Poespoprodjo JR, Fobia W, Kenangalem E, Lampah DA, Warikar N, Seal A, McGready R, Sugiarto P, Tjitra E, Anstey NM, Price RN (2008). Adverse pregnancy outcomes in an area where multidrugresistant Plasmodium vivax and Plasmodium falciparum infections are endemic. Clin Infect Dis.46:1374-81.

30. Population Services International (PSI) (2017). Transforming the Private Sector to Support Universal Malaria Diagnostic Coverage Lessons learned from Kenya, Madagascar and Tanzania. Washington, DC.

31. President's Malaria Initiative (2016). Malaria Operational Plan FY2016.

32. Rogerson SJ, Desai M, Mayor A, Sicuri E, Taylor SM, van Eijk AM (2018). Burden, pathology, and costs of malaria in pregnancy: new developments for an old problem. Lancet Infect Dis

33. Steketee RW, Nahlen BL, Parise ME, Menéndez C (2001). The burden of malaria in pregnancy in malaria-endemic areas. Am J Trop Med Hyg; 64 (suppl): 28

34. Strom GE, Haanshuus CG, Fataki M, Langeland N, Blomberg B (2013). Challenges in diagnosing paediatric malaria in Dar es Salaam, Tanzania. Malar J. 12:228.

35. Umbers AJ, Aitken EH, Rogerson SJ (2011). Malaria in pregnancy: small babies, big problem. Trend Parasitol. 27:168-75.

36. Wilson ML (2013). Laboratory diagnosis of malaria: conventional and rapid diagnostic methods. Arch Pathol Lab Med. 137:805-11.

37. World Health Organization (2006). Guidelines for the Treatment of malaria. Geneva: World Health Organization.

38. World Health Organization (2012) Universal access to malaria diagnostic testing. Geneva: World Health Organization

39. World Health Organization (2014). Recommendation for malaria diagnostics in low transmission settings. Geneva: World Health Organization.

40. World Health Organization (2015). World Malaria Report. Geneva: World Health Organization. 